\title{
An efficient algorithm for simulating scattering by a large number of two dimensional particles
}

\author{
Mahadevan Ganesh $^{1} \quad$ Stuart C. Hawkins ${ }^{2}$
}

(Received 31 January 2011; revised 10 May 2011)

\begin{abstract}
Simulation of waves scattered by a large number of particles is important for several applications - for example, to investigate interactions between particles in an ensemble - and hence to design efficient configurations. Substantial computer memory is required for the direct treatment of an ensemble with hundreds of particles as a single scattering configuration. This memory bottleneck is avoided by using multiple scattering iterative methods, which allow treatment of one particle at a time, but require substantial computing time at each step of the iteration to take into account reflections from the rest of the particles, and require a large number of iterations for convergence. We develop a novel fast, high order, memory efficient algorithm to simulate multiple acoustic scattering induced by an ensemble with hundreds of particles in two space dimensions.
\end{abstract}

http://anziamj . austms.org.au/ojs/index.php/ANZIAMJ/article/view/3954 gives this article, (c) Austral. Mathematical Soc. 2011. Published May 16, 2011. IssN 1446-8735. (Print two pages per sheet of paper.) Copies of this article must not be made otherwise available on the internet; instead link directly to this URL for this article. 


\section{Contents}

1 Introduction

C140

2 A multiple acoustic scattering model

C142

3 A fast high order iterative algorithm

C144

4 Numerical Results

C150

References

C154

\section{Introduction}

The understanding of many physical phenomena and processes is enhanced by simulating the multiple scattering of waves by configurations of many particles. Books by Martin [8] and Mischenko et al. [9] and references therein describe various computational and mathematical aspects of multiple scattering configurations and applications. We are interested in two dimensional configurations consisting of hundreds of particles, such as those in Figure 1, which naturally occur in various applications $[8,9]$, with the diameter of each particle at least one wavelength.

Recently there has been substantial research activity to generalise various single obstacle exterior scattering algorithms to the multiple particle case [1, $2,5,6]$. One direct approach is to treat multiple particle acoustic scattering by considering the multiple particle system as a single configuration and solving coupled discretised systems [1]. This approach was demonstrated for two dimensional problems using a few configurations, with each configuration consisting of at most three particles [1]. While such an approach is expedient, the computer memory required for configurations consisting of hundreds of particles is prohibitive. 
The mathematical framework of the boundary decomposition technique [3] is sometimes used to remove the memory bottleneck by iteratively decomposing the multiple scattering problem into several single obstacle scattering problems, taking into account at each step of the iteration reflected waves from other particles in the configuration. This Neumann iterative technique was used for acoustic and electromagnetic scattering in two and three dimensions [2, 5, 6]. Acoustic scattering by only two obstacles has been demonstrated [2, 5], but other work demonstrated that, even for two obstacles, the Neumann iterations diverge when the obstacles are close together [6]. Hence the Neumann-type iterative methods are not appropriate to simulate scattering by hundreds of obstacles.

Although iterative methods facilitate substantial memory reduction for scattering by configurations with a large number of particles, the computational time for such methods is prohibitively large. This is mainly because, at each step of the iteration and for each particle in the configuration, reflected waves from all of the other particles need to be evaluated in order to formulate the associated single obstacle scattering problem. Such a formulation requires the evaluation of thousands of boundary integrals at each step of the iteration, leading to several days or weeks of simulation for multiple scattering configurations such as those in Figure 1. Because of this computational burden, scattering by large numbers of particles has attracted minimal attention [9].

We overcome this computational bottleneck by developing a novel fast high order memory efficient algorithm to simulate multiple acoustic scattering by an ensemble with hundreds of particles in two space dimensions. Our iterative approach is suitable for large particle configurations and avoids evaluation of thousands of boundary integrals to compute the reflected waves at each step of the iteration.

In the next section we introduce the multiple scattering problem and describe its reformulation as a boundary integral equation. Section 3 describes our fast high order memory efficient fully discrete Galerkin iterative algorithm to simulate scattering from configurations with a large number of particles. 


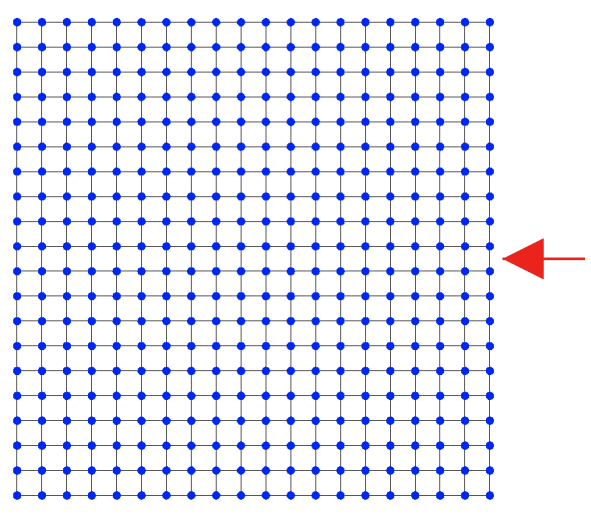

FIGURE 1: A multiple scattering configuration with 400 particles in a lattice.

Finally, Section 4 demonstrates the efficiency of our multiple scattering algorithm by simulating scattering from configurations such as the one in Figure 1, consisting of several hundred convex or non-convex particles.

\section{$2 \quad$ A multiple acoustic scattering model}

The spatial component $u$ of the time harmonic acoustic field scattered in two space dimensions by a large number of closed obstacles $D_{1}, \ldots, D_{M} \subset \mathbb{R}^{2}$ located in a homogeneous medium satisfies the Helmholtz equation $[4,8,9]$

$$
\triangle \mathfrak{u}(\boldsymbol{x})+k^{2} u(x)=0, \quad x \in \mathbb{R}^{2} \backslash \bigcup_{J=1}^{M} \overline{\mathrm{D}_{J}},
$$

where $k=2 \pi / \lambda$ is the wavenumber and $\lambda$ is the wavelength, and the radiation condition

$$
\lim _{|x| \rightarrow \infty} \sqrt{|x|}\left(\frac{\partial u}{\partial x}-i k u\right)=0
$$


uniformly in all directions. The behaviour of the scattered field a long way from a scatterer is described by the far field

$$
u^{\infty}(\widehat{x})=\lim _{|x| \rightarrow \infty} \sqrt{|x|} e^{-i k|x|} u(x), \quad \widehat{x}=\frac{x}{|x|} .
$$

The far field is important for acoustic cross section and inverse scattering computations, and we use this physical quantity in our novel fast algorithm. The scattered field is induced by an incident plane wave $\mathfrak{u}^{\mathrm{inc}}(\boldsymbol{x})=e^{\mathbf{i k x} \cdot \widehat{\mathbf{d}}}$ with direction given by the unit vector $\widehat{\mathbf{d}}$. For brevity of presentation, we assume that the obstacles $D_{1}, \ldots, D_{N} \subset \mathbb{R}^{3}$ are sound-soft. Thus the total field $u+u^{\text {inc }}$ vanishes on the surface of each scatterer, leading to the boundary condition

$$
u(x)=-u^{\text {inc }}(x), \quad x \in \cup_{I=1}^{M} \partial D_{I},
$$

where $\partial D_{I}$ denotes the surface of $D_{I}$ for $I=1, \ldots, M$.

Due to the unbounded domain $\mathbb{R}^{2} \backslash \bigcup_{\mathrm{J}=1}^{\mathrm{M}} \overline{\mathrm{D}_{\mathrm{J}}}$ in (1), it is efficient to reformulate the scattering problem using a boundary integral representation of the scattered field that automatically satisfies the radiation condition (2). Such a representation requires computation of $M$ acoustic density functions $\phi_{J}$ for $J=1, \ldots, M$. The density functions are defined on the boundaries of the particles in the configuration and satisfy a system of boundary integral equations. The disadvantage of this favourable reformulation is that any discretisation process requires solution of a dense complex linear system. This is independent of whether compactly supported local basis functions or global basis functions are used in the discretisation process.

Coupling of the single and double layer operators, with coupling parameter $\eta>$ 0 , is necessary to obtain a uniquely solvable boundary integral reformulation of the acoustic scattering problem [4]. (In our implementation we take $\eta=k$, which minimises the condition number of the boundary integral operators [4].) Using the fundamental solution of the two dimensional Helmholtz equation,

$$
\Phi(\boldsymbol{x}, \mathbf{y})=\frac{i}{4} \mathrm{H}_{0}^{(1)}(k|x-\mathbf{y}|)
$$


where $H_{0}^{(1)}$ is the Hankel function of the first kind, we represent the exterior field $\boldsymbol{u}$ satisfying (1)-(2) as

$u(x)=\sum_{J=1}^{M} \int_{\partial D_{J}}\left\{\frac{\partial \Phi}{\partial \mathfrak{n}(\boldsymbol{y})}(\boldsymbol{x}, \boldsymbol{y})-i \eta \Phi(\boldsymbol{x}, \boldsymbol{y})\right\} \phi_{\mathrm{J}}(\mathbf{y}) \mathrm{ds}(\mathbf{y}), \quad \boldsymbol{x} \in \mathbb{R}^{2} \backslash \bigcup_{\mathrm{I}=1}^{M} \overline{\mathrm{D}_{\mathrm{I}}}$

Using the boundary condition (4), the $M$ unknown acoustic densities $\phi_{\mathrm{J}}$ for $J=1, \ldots, M$, satisfy a system of $M$ uniquely solvable boundary integral equations $[4,8]$

$\phi_{\mathrm{I}}(\boldsymbol{x})+\sum_{J=1}^{M}\left(\mathcal{K}_{\mathrm{IJ}} \phi_{\mathrm{J}}-i \eta \mathcal{S}_{\mathrm{IJ}} \phi_{\mathrm{J}}\right)(\boldsymbol{x})=-2 \mathrm{u}^{\mathrm{inc}}(\boldsymbol{x}), \quad \boldsymbol{x} \in \partial \mathrm{D}_{\mathrm{I}}, \quad \mathrm{I}=1, \ldots, \mathrm{M}$,

where, for $\mathrm{I}, \mathrm{J}=1, \ldots, M$, the operators $\mathcal{K}_{\mathrm{IJ}}$ and $\mathcal{S}_{\mathrm{IJ}}$ are respectively the acoustic double and single layer operators with source density defined on the Jth obstacle and observation point on the Ith obstacle,

$$
\mathcal{K}_{\mathrm{IJ}} v(\boldsymbol{x})=2 \int_{\partial \mathrm{D}_{\mathrm{J}}} \frac{\partial \Phi}{\partial \mathfrak{n}(\mathbf{y})}(\boldsymbol{x}, \mathbf{y}) v(\mathbf{y}) \mathrm{ds}(\mathbf{y}), \quad \boldsymbol{x} \in \partial \mathrm{D}_{\mathrm{I}}, \quad \mathrm{I}, \mathrm{J}=1, \ldots, \mathrm{M}
$$

and

$$
\left(\mathcal{S}_{\mathrm{IJ}} v\right)(\boldsymbol{x})=2 \int_{\partial \mathrm{D}_{\mathrm{J}}} \Phi(\boldsymbol{x}, \boldsymbol{y}) \mathcal{v}(\mathbf{y}) \mathrm{ds}(\mathbf{y}), \quad \boldsymbol{x} \in \partial \mathrm{D}_{\mathrm{I}}, \quad \mathrm{I}, \mathrm{J}=1, \ldots, \mathrm{M} .
$$

These operators are weakly singular when $\mathrm{I}=\mathrm{J}$ and smooth otherwise.

\section{A fast high order iterative algorithm}

For each $J=1, \ldots, M$, we parametrize the closed boundary curve $\partial D_{J}$ of the scatterer $D_{J}$ using the $2 \pi$-periodic mapping

$$
\mathbf{q}^{\mathrm{J}}(\theta)=\left(\mathbf{q}_{1}^{J}(\theta), \mathbf{q}_{2}^{J}(\theta)\right), \quad \theta \in[0,2 \pi] .
$$


Using this parametrisation we write $\mathbf{y}=\mathbf{q}^{\mathrm{J}}(\mathbf{s})$ and $\boldsymbol{x}=\mathbf{q}^{\mathrm{I}}(\mathbf{t})$. Then (7)-(8) give

$$
\begin{aligned}
& \left(\mathcal{K}_{\mathrm{IJ}} v\right)\left(\mathbf{q}^{\mathrm{I}}(\mathrm{t})\right)=\int_{0}^{2 \pi} \mathrm{L}^{\mathrm{IJ}}(\mathrm{t}, \mathrm{s}) v\left(\mathbf{q}^{\mathrm{J}}(\mathrm{s})\right) \mathrm{ds}, \quad \mathrm{I}, \mathrm{J}=1, \ldots, \mathrm{M}, \\
& \left(\mathcal{S}_{\mathrm{IJ}} v\right)\left(\mathbf{q}^{\mathrm{I}}(\mathrm{t})\right)=\int_{0}^{2 \pi} M^{\mathrm{IJ}}(\mathrm{t}, \mathrm{s}) v\left(\mathbf{q}^{\mathrm{J}}(\mathrm{s})\right) \mathrm{d} s, \quad \mathrm{I}, \mathrm{J}=1, \ldots, M,
\end{aligned}
$$

where

$$
\begin{aligned}
\mathrm{L}^{\mathrm{I}}(\mathrm{t}, \mathrm{s}) & =2 \frac{\partial \Phi}{\partial \mathbf{n}(\mathbf{y})}\left(\mathbf{q}^{\mathrm{I}}(\mathrm{t}), \mathbf{q}^{\mathrm{J}}(\mathrm{s})\right) \mathcal{J}^{\mathrm{J}}(\mathrm{s}), \quad \mathrm{I}, \mathrm{J}=1, \ldots, \mathrm{M}, \\
\mathrm{M}^{\mathrm{IJ}}(\mathrm{t}, \mathrm{s}) & =2 \Phi\left(\mathbf{q}^{\mathrm{I}}(\mathbf{t}), \mathbf{q}^{\mathrm{J}}(\mathrm{s})\right) \mathcal{J}^{\mathrm{J}}(\mathrm{s}), \quad \mathrm{I}, \mathrm{J}=1, \ldots, \mathrm{M},
\end{aligned}
$$

where $\mathcal{J}^{J}$ is the Jacobian of $\mathbf{q}^{J}$ for $J=1, \ldots, M$.

The boundary integral equation (6) becomes

$$
\psi_{\mathrm{I}}+\sum_{J=1}^{M} \mathcal{M}_{\mathrm{IJ}} \psi_{\mathrm{J}}=\mathrm{f}_{\mathrm{I}}, \quad \psi_{\mathrm{I}} \in \mathcal{C}([0,2 \pi]), \quad \mathrm{I}=1, \ldots, M
$$

where

$$
\begin{aligned}
& \psi_{\mathrm{I}}(\mathrm{t})=\phi_{\mathrm{I}}\left(\mathbf{q}^{\mathrm{I}}(\mathrm{t})\right), \quad \mathrm{f}_{\mathrm{I}}(\mathrm{t})=-2 \mathrm{u}^{\mathrm{inc}}\left(\mathbf{q}^{\mathrm{I}}(\mathrm{t})\right), \quad \mathrm{t} \in[0,2 \pi], \\
& \left(\mathcal{M}_{\mathrm{IJ}} w\right)(\mathrm{t})=\int_{0}^{2 \pi}\left[\mathrm{L}^{\mathrm{IJ}}(\mathrm{t}, \mathrm{s})-i \eta M^{\mathrm{IJ}}(\mathrm{t}, \mathrm{s})\right] w(\mathrm{~s}) \mathrm{ds}, \quad \mathrm{t} \in[0,2 \pi],
\end{aligned}
$$

and $\mathcal{C}([0,2 \pi])$ is the space of all continuous $2 \pi$-periodic functions. The smoothness of $\psi_{\mathrm{I}}$, for $\mathrm{I}=1, \ldots, M$, is determined by the smoothness of the particle $\mathrm{D}_{\mathrm{I}}$ in the $\mathrm{M}$-particle multiple scattering configuration.

We must approximate the $M$ acoustic densities $\psi_{\mathrm{I}} \in \mathcal{C}([0,2 \pi])$ in some appropriate finite dimensional subspaces of $\mathfrak{C}([0,2 \pi])$. We seek approximations $\psi_{\mathrm{I}, \mathrm{N}}$ to $\psi_{\mathrm{I}}$, for $\mathrm{I}=1, \ldots, M$, in an $\mathcal{O}(\mathrm{N})$-dimensional subspace $\mathbb{V}_{N}$ of $\mathcal{C}([0,2 \pi])$. (For notational convenience, we allow the discretisation parameter $\mathrm{N}$ to be 
independent of the particle index I.) The $M$ coupled boundary integral equations on an infinite dimensional space are then replaced by

$$
\psi_{\mathrm{I}, \mathrm{N}}+\sum_{J=1}^{M} \mathcal{M}_{\mathrm{IJ}} \psi_{\mathrm{J}, \mathrm{N}}=\mathbb{P}_{\mathrm{N}} \mathrm{f}_{\mathrm{I}}, \quad \psi_{\mathrm{I}, \mathrm{N}} \in \mathbb{V}_{\mathrm{N}}, \quad \mathrm{I}=1, \ldots, \mathrm{M},
$$

where $\mathbb{P}_{N} f_{I}$ is the orthogonal projection of $f_{I}$ onto $\mathbb{V}_{N}$. For a fixed positive integer $\alpha$ (independent of $\mathrm{N}$ ), let

$$
\mathbb{V}_{N}=\operatorname{span}\left\{Y_{n}: n=0, \ldots, \alpha N\right\}
$$

be the finite dimensional approximation space.

Remark 1 In our implementation, for various values of $N$ (that depend on the shape and acoustic diameter of the particles, that is, the product of the wavenumber with the diameter of the particles) we choose $\alpha=2$ and $Y_{n}(s)=e^{i(n-N) s} / \sqrt{2 \pi}$ for $n=0, \ldots, \alpha N$ and $s \in[0,2 \pi]$ so that $\mathbb{V}_{N}$ is the space of all trigonometric polynomials of degree at most $\mathrm{N}$.

Such a choice ensures that $\mathbb{P}_{N} f_{I}$ is a high order approximation of $f_{I}$ and that high order approximations $\psi_{\mathrm{I}, \mathrm{N}}$ to $\psi_{\mathrm{I}}$ exist in the space $\mathbb{V}_{\mathrm{N}}$. It is efficient to exploit such high order approximations, especially when each particle in the multiple configuration is sufficiently smooth. Such a choice is also very competitive with the boundary element discretisation (which requires at least ten points per wavelength, per obstacle, for two digit accuracy) for the multiple scattering problem with non-smooth particle configurations.

For $\psi_{\mathrm{I}, \mathrm{N}} \in \mathbb{V}_{\mathrm{N}}$ we use (11) to write

$$
\psi_{\mathrm{I}, \mathrm{N}}=\sum_{n=0}^{\alpha N} x_{I, n} Y_{n}, \quad I=1, \ldots, M .
$$

The $\mathcal{O}(M N)$ coefficients in (12) for the $M$ approximate acoustic densities satisfying the $M$ coupled boundary integral equations (10) are sometimes 
computed by solving the $\mathcal{O}(\mathrm{MN}) \times \mathcal{O}(\mathrm{MN})$ dense complex linear Galerkin system

$$
\left\langle\psi_{I, N}, Y_{n}\right\rangle+\sum_{J=1}^{M}\left\langle\mathcal{M}_{I J} \psi_{J, N}, Y_{n}\right\rangle=\left\langle f_{I}, Y_{n}\right\rangle, \quad n=0, \ldots, \alpha N, \quad I=1, \ldots, M
$$

where $\langle\cdot, \cdot\rangle$ is the standard $\mathrm{L}_{2}$-inner product on $[0,2 \pi]$. Such a direct approach, with an $\mathcal{O}(M N) \times \mathcal{O}(M N)$ dense complex matrix, is not memory efficient (and is prohibitive in standard contemporary systems with say 4-8 GB memory), especially when $M$ is large. Using the mathematical boundary decomposition framework [3] removes this memory bottleneck,

In particular, the iterative boundary decomposition method, or Neumann iterative method, requires at each iteration solution of only $M$ dense complex linear systems (one for each particle) with each system of size $\mathcal{O}(N) \times \mathcal{O}(N)$. This removes the memory bottleneck.

For any fixed discretisation parameter $\mathrm{N}$ we compute approximations $\psi_{\mathrm{I}, \mathrm{N}}^{(\ell)}$ to the solutions $\psi_{\mathrm{I}, \mathrm{N}}$ of $(13)$ for each $\mathrm{I}=1, \ldots, M$ and for $\ell=1, \ldots, \mathrm{L}$ as follows. For $\ell=1$, the initial approximations are computed by assuming each particle in the configuration is independent of all of the other particles, leading to an $\mathcal{O}(\mathrm{N}) \times \mathcal{O}(\mathrm{N})$ dense complex linear system for each unknown $\psi_{I, N}^{(1)} \in \mathbb{V}_{N}$ for $I=1, \ldots, M$,

$$
\left\langle\psi_{\mathrm{I}, \mathrm{N}}^{(1)}, Y_{n}\right\rangle+\left\langle\mathcal{M}_{\mathrm{II}} \psi_{\mathrm{I}, \mathrm{N}}^{(1)}, Y_{n}\right\rangle=\left\langle\mathrm{f}_{\mathrm{I}}, Y_{n}\right\rangle, \quad \mathrm{n}=0, \ldots, \alpha \mathrm{N}, \quad \mathrm{I}=1, \ldots, M .
$$

We solve each of these $\mathcal{O}(\mathrm{N}) \times \mathcal{O}(\mathrm{N})$ systems (14) to high order accuracy.

The main task required to efficiently solve (14) is to discretize the $M$ weakly singular boundary integrals $\mathcal{M}_{\mathrm{II}} \mathcal{w}$, for $\mathrm{I}=1, \ldots, M$, for a given density $w$, with high order accuracy. This is achieved by splitting the kernels $\mathrm{L}^{\mathrm{II}}$ and $\mathrm{M}^{\mathrm{II}}$ in $\mathcal{M}_{\text {II }}$ into weakly singular and smooth parts respectively

$$
\mathrm{L}^{\mathrm{II}}(\mathrm{t}, \mathrm{s})=\mathrm{L}_{1}^{\mathrm{II}}(\mathrm{t}, \mathrm{s}) \log \left(4 \sin ^{2} \frac{\mathrm{t}-\mathrm{s}}{2}\right)+\mathrm{L}_{2}^{\mathrm{II}}(\mathrm{t}, \mathrm{s})
$$


and

$$
M^{\mathrm{II}}(\mathrm{t}, \mathrm{s})=\mathrm{M}_{1}^{\mathrm{II}}(\mathrm{t}, \mathrm{s}) \log \left(4 \sin ^{2} \frac{\mathrm{t}-\mathrm{s}}{2}\right)+\mathrm{M}_{2}^{\mathrm{II}}(\mathrm{t}, \mathrm{s})
$$

with analytic kernels $\mathrm{L}_{1}^{\mathrm{II}}, \mathrm{L}_{2}^{\mathrm{II}}, \mathrm{M}_{1}^{\mathrm{II}}, \mathrm{M}_{2}^{\mathrm{II}}$, for $\mathrm{I}=1, \ldots, \mathrm{M}$, having representations in a classical form [4, p. 68]. In particular, the resulting $2 \pi$-periodic integrals are of the form $\int_{0}^{2 \pi} g(s) \log \left(4 \sin ^{2}(t-s) / 2\right) d s$ and $\int_{0}^{2 \pi} g(s) d s$ for given smooth $2 \pi$-periodic functions $\mathrm{g}$. Utilising interpolatory approximations $g_{N}$ in the space $Y_{N}$ described in Remark 1 , at $2 N+1$ equally spaced points in $[0,2 \pi)$, leads to quadrature approximations $M_{I I, N}$ of the boundary integrals $M_{I I}$ in (14) with high order accuracy [4]. We also use this quadrature rule to discretize the $\mathrm{L}_{2}$-inner product $\langle\cdot, \cdot\rangle$ in (14) to high order accuracy, and the associated discretisation is denoted by $\langle\cdot, \cdot\rangle_{\mathrm{N}}$.

Thus a physically meaningful initial approximation, in any iterative boundary decomposition method for the multiple scattering problem, is obtained by solving $M$ fully discrete $\mathcal{O}(N) \times \mathcal{O}(N)$ algebraic systems

$$
\left\langle\left(\mathcal{J}+\mathcal{M}_{\mathrm{II}, \mathrm{N}}\right) \psi_{\mathrm{I}, \mathrm{N}}^{(1)}, Y_{\mathrm{n}}\right\rangle_{\mathrm{N}}=\left\langle\mathrm{f}_{\mathrm{I}}, Y_{\mathrm{n}}\right\rangle_{\mathrm{N}}, \quad \mathrm{n}=0, \ldots, \alpha \mathrm{N}, \quad \mathrm{I}=1, \ldots, M .
$$

If required, the LU-decomposition of each of the $M$ complex dense matrices, each of size $\mathcal{O}(N) \times \mathcal{O}(N)$, can be computed once and stored. On parallel architectures this can be done in a distributed way, exploiting memory across multiple platforms. Storing the LU-factors in this way is useful for efficient computation at subsequent iterations of $\psi_{\mathrm{I}, \mathrm{N}}^{(\ell)}$ for $\ell=2, \ldots, \mathrm{L}$. Here $\mathrm{L}$ denotes the maximum number of iterates before the stopping criterion is met.

The subsequent iterations require solution of the $\mathcal{O}(N) \times \mathcal{O}(N)$ dense complex linear systems such as the Neumann iterates $[2,5,6]$

$$
\left\langle\left(\mathcal{J}+\mathcal{M}_{I I, N}\right) \psi_{I, N}^{(\ell)}, Y_{n}\right\rangle_{N}=\left\langle f_{I}, Y_{n}\right\rangle_{N}-\sum_{J=1, J \neq I}^{M}\left\langle\mathcal{M}_{I J} \psi_{J, N}^{(\ell-1)}, Y_{n}\right\rangle_{N}
$$

for $\ell=1, \ldots, \mathrm{L}, \mathrm{n}=0, \ldots, \alpha \mathrm{N}$, and $\mathrm{I}=1, \ldots, \mathrm{M}$. Although the boundary decomposition removes the memory bottleneck, we observed that in many 
cases the Neumann iterations in (16) do not converge, even after several days of computations (with $L>1000$ ). This is not unexpected; divergence has been demonstrated for scattering by even two nearby obstacles [6]. Indeed the theory imposes separation conditions on the particles that are not feasible for configurations consisting of hundreds of particles [3].

An alternative way to view the iterative solver is to recognize that the Neumann-type iterates in (16) are equivalent to iterates of a block Jacobi method. Thus a natural, but unexplored, next step is to utilize Krylov subspace methods and consider a multiple scattering equivalent of the Generalized Minimal Residual (GMREs) method. Although we have observed that this approach is superior to the Neumann-type iterates, the number L of GMRES iterations is still prohibitively large. We overcome this problem by developing a multiple scattering version of the FGMRES (Flexible-GMRES) method so that for configurations in Figure 1, we achieve convergence with the number of iterations being fewer than ten percent of the number of unknowns.

To set up the right hand side vectors in (16) at each iteration, the iterative method must compute the $\mathcal{O}\left(M^{2}\right)$ boundary integrals $\sum_{\mathrm{J}=1, \mathrm{~J} \neq \mathrm{I}}^{M} \mathcal{M}_{\mathrm{IJ}} \psi_{\mathrm{J}, \mathrm{N}}^{(\ell-1)}$ for $\mathrm{I}=1, \ldots, M$ at $\mathcal{O}(\mathrm{N})$ points. The algorithm that uses a multiple scattering version of FGMRES and evaluates these $\mathcal{O}\left(M^{2} N L\right)$ boundary integrals using the high order quadrature rule is denoted the FGMRES-INT algorithm.

The $M^{2}$ factor in the number of boundary integrals makes the FGMRESINT algorithm computationally expensive when $M$ is large. We propose a new approach to reduce the $M^{2}$ factor to just $M$, that is, to evaluate just $\mathcal{O}(\mathrm{MNL})$ boundary integrals. The result is a faster version of the FGMRES-INT algorithm that we denote the FGMRES-FAST algorithm. In the next section we numerically demonstrate the advantage of the FGMRES-FAST algorithm compared to the FGMRES-INT algorithm.

A full description of the FGMRES-FAST algorithm and its convergence analysis is beyond the scope of this article, but we briefly explain the main idea. For each $I, J=1, \ldots, M$ with $I \neq J$, the integral $\mathcal{M}_{I J} \psi_{J, N}^{(\ell-1)}(x)$ for $x \in \partial D_{I}$ represents the scattered field on the Ith obstacle induced by the surface current 
from the Jth obstacle. Each of these $\mathcal{O}\left(\mathrm{M}^{2}\right)$ scattered fields is represented as a linear combination of classical wave functions [4]. This representation is related to the T-matrix method $[8,9]$. While the standard T-matrix approach to compute the coefficients of the linear combination is not numerically stable, we recently proposed and demonstrated a numerically stable way to compute the coefficients using the far field [7]. Such coefficients are computed by evaluating only $\mathcal{O}(M)$ boundary integrals.

\section{$4 \quad$ Numerical Results}

We demonstrate the efficiency of the FGMRES-INT and FGMRES-FAST algorithms for simulating scattering by large numbers of particles. Our experiments show that, in all cases, FGMRES-FAST is several times faster than FGMRES-INT. All of our experiments are performed in Matlab on a $2.0 \mathrm{GHz}$ Opteron processor.

In our implementation the outer FGMRES iteration is preconditioned using a nearest neighbours preconditioner, which is implemented approximately using three steps of block Jacobi preconditioned GMRES. This choice provides the optimal balance between conditioning and $\mathrm{CPU}$ time to implement the preconditioner. The FGMRES iteration is continued (without restarting) until the residual norm is reduced by a factor of $10^{-8}$.

Our test configurations consist of unit circles or peanut shaped particles. The unit circle is a standard test scatterer for both single and multiple scattering. The peanut is a challenging scatterer because, as shown in Figure 3, it is non-convex. In all of our experiments the incident wavelength is such that the scatterers have diameter one wavelength. For these test scatterers we use $\mathrm{N}=10$ and $\mathrm{N}=35$ respectively. We verified numerically that these choices lead to a relative error less than $10^{-7}$ for single scattering problems.

Table 1 (left) tabulates the CPU time (in minutes) required to solve the linear system for scattering by $M$ circles each of diameter one wavelength 
TABLE 1: CPU time (minutes) for solving the linear system to simulate multiple scattering by $M$ circles of diameter one wavelength using $N=10$ (left) and by $M$ peanut shaped particles each of diameter one wavelength using $\mathrm{N}=35$ (right).

\begin{tabular}{rrrrr}
\hline & \multicolumn{2}{c}{$M$ circles } & \multicolumn{2}{c}{$M$ peanuts } \\
$M$ & FGMRES-INT & FGMRES-FAST & FGMRES-INT & FGMRES-FAST \\
\hline 25 & 4.5 & 0.5 & 13.1 & 0.6 \\
49 & 27.7 & 2.9 & 76.2 & 3.4 \\
100 & 191.8 & 19.3 & 525.6 & 22.3 \\
225 & 2422.9 & 245.7 & 6157.0 & 257.9 \\
400 & 13664.7 & 1397.3 & 33879.1 & 1380.1 \\
\hline
\end{tabular}

arranged in a square lattice. In each of these experiments the high order algorithm facilitates a low 21-dimensional ansatz space. For this problem the FGMRES-FAST method is about ten times faster than the FGMRES-INT method.

Table 1 (right) tabulates the CPU time (in minutes) required to solve the linear system for $M$ peanuts of diameter one wavelength arranged in a square lattice. The dimension of the ansatz space in each of these experiments using the high order algorithm is only 71. For this problem the FGMRES-FAST method requires less than $5 \%$ of the CPU time compared to that by FGMRES-INT method.

Finally, Figure 2 visualizes the bistatic acoustic cross section of 400 circles of diameter one wavelength, computed using the FGMRES-FAST method with $\mathbf{N}=10$. The acoustic cross section, in decibels, is

$$
\sigma_{N}=10 \log _{10} 2 \pi\left|u_{N}^{\infty}\right|^{2}
$$

where $u_{N}^{\infty}$ is the approximation to the far field of the ensemble, computed from $\psi_{\mathrm{J}, \mathrm{N}}$ for $\mathrm{J}=1, \ldots, \mathrm{N}$. Figure 3 visualizes the bistatic acoustic cross section of 400 peanut shaped particles of diameter one wavelength, arranged in a square lattice, computed using FGMRES-FAST with $\mathrm{N}=35$. 


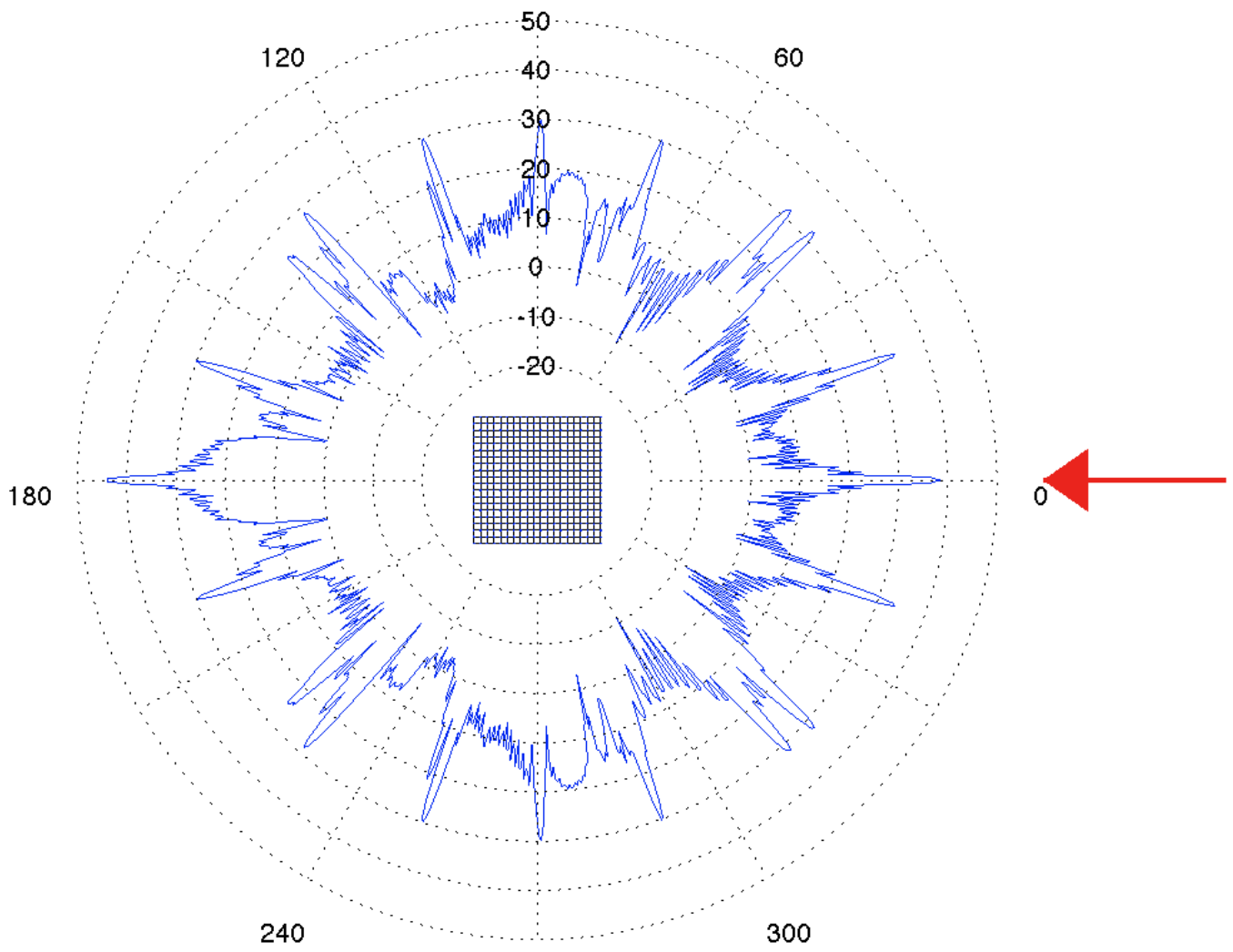

Figure 2: Bistatic acoustic cross section (in dB) of 400 circles of diameter one wavelength computed using FGMRES-FAST and $\mathrm{N}=10$. 


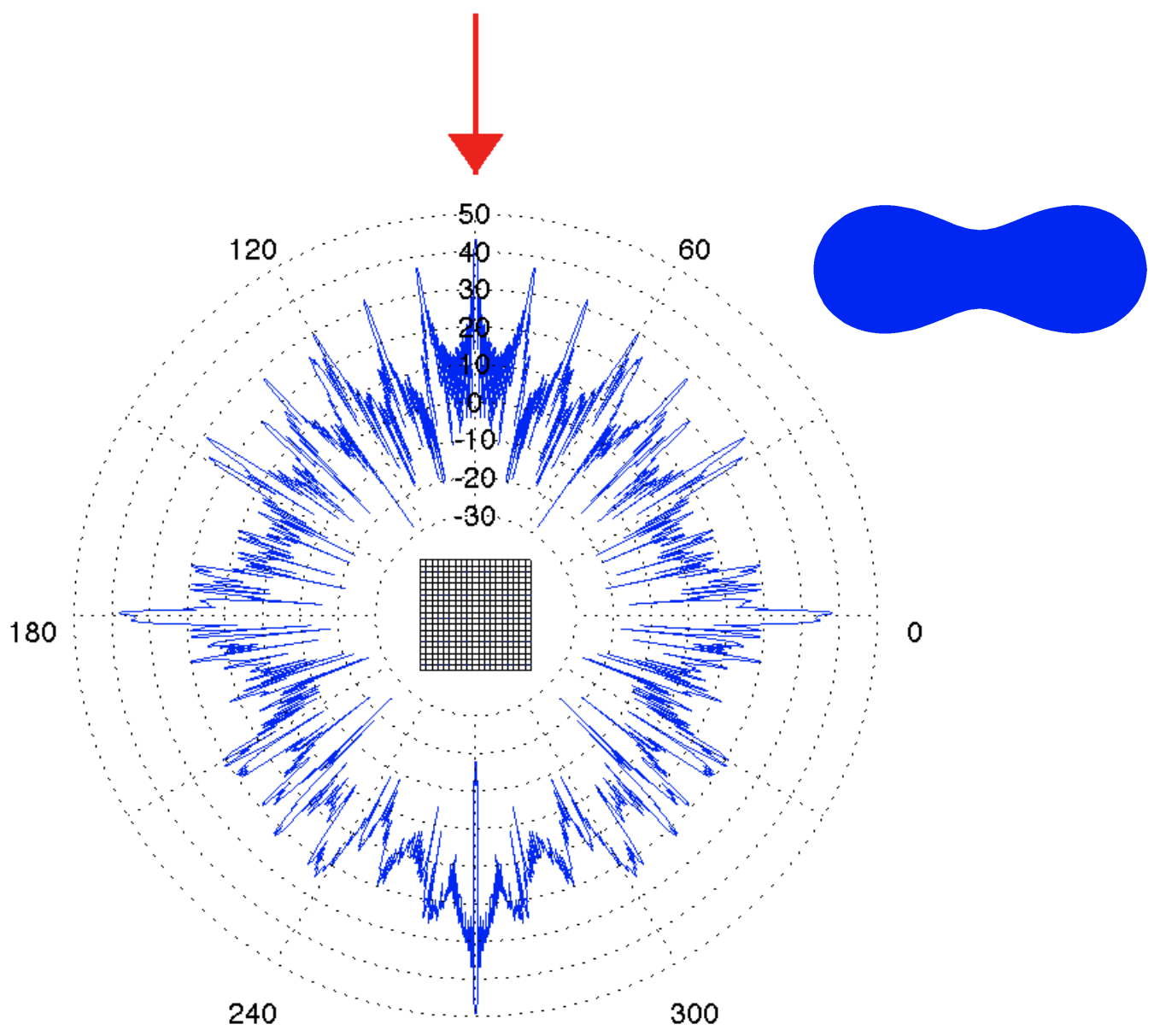

Figure 3: Bistatic acoustic cross section (in dB) of 400 peanuts of diameter one wavelength computed using FGMRES-FAST and $N=35$. (The inset shows the shape of the peanut scatterer and the incident direction.) 


\section{References}

[1] S. Acosta and V. Villamizar. Coupling of Dirichlet-to-Neumann boundary condition and finite difference methods in curvilinear coordinates for multiple scattering. J. Comput. Phys., 229:5498-5517, 2010. doi:10.1016/j.jcp.2010.04.011. C140

[2] A. Anand, Y. Boubendir, F. Ecevit, and F. Reitich. Analysis of multiple scattering iterations for high-frequency scattering problems II: The three-dimensional scalar case. Numer. Math., 114:373-427, 2010. doi:10.1007/s00211-009-0263-1. C140, C141, C148

[3] M. Balabane. Boundary decomposition for Helmholtz and Maxwell equations 1: disjoint sub-scatterers. Asymp. Anal., 38:1-10, 2004. http://iospress.metapress. com/content/vu2bd0w9mkem8966/. C141, C147, C149

[4] D. Colton and R. Kress. Inverse Acoustic and Electromagnetic Scattering Theory. Springer, 1998. C142, C143, C144, C148, C150

[5] F. Ecevit and F. Reitich. Analysis of multiple scattering iterations for high-frequency scattering problems. I: The two-dimensional case. Numer. Math., 114:271-354, 2009. doi:10.1007/s00211-009-0249-z. C140, C141, C148

[6] M. Ganesh and S. C. Hawkins. A high-order algorithm for multiple electromagnetic scattering in three dimensions. Numer. Algorithms, 50:469-510, 2009. doi:10.1007/s11075-008-9238-z. C140, C141, C148, C149

[7] M. Ganesh and S. C. Hawkins. Three dimensional electromagnetic scattering T-matrix computations. J. Comp. Appl. Math., 234:1702-1709, 2010. doi:10.1016/j.cam.2009.08.018. C150 
[8] P. A. Martin. Multiple Scattering: Interaction of Time-Harmonic Waves with $N$ Obstacles. Cambridge University Press, 2006. C140, C142, C144, $\mathrm{C} 150$

[9] M. I. Mishchenko, L. D. Travis, and A. A. Lacis. Multiple Scattering of Light by Particles: Radiative Transfer and Coherent Backscattering. Cambridge University Press, 2006. C140, C141, C142, C150

\section{Author addresses}

1. Mahadevan Ganesh, Department of Mathematical and Computer Sciences, Colorado School of Mines, Golden, CO 80401, UsA. mailto:mganesh@mines . edu

2. Stuart C. Hawkins, Department of Mathematics, Macquarie University, Sydney, NSW 2109, Australia.

mailto:stuart.hawkins@mq.edu . au 